\title{
Problemas de la extensión rural en América Latina
}

\section{Fernando Landini*}

Perfiles Latinoamericanos, 24(47)

2016 | pp. 47-68

DOI: $10.18504 / \mathrm{pl} 2447-005-2016$

\section{Resumen}

Para contribuir al conocimiento de los problemas que enfrentan los extensionistas rurales en América Latina, se realizó una encuesta de preguntas abiertas a técnicos que trabajan en diez países diferentes. Las preguntas se categorizaron según los principios de la teoría fundamentada. Luego, las diferencias entre las muestras nacionales se estudiaron utilizando chi cuadrado. Se detectaron quince problemas con un promedio destacado de referencias resaltando tres: dificultades con el trabajo grupal/asociativo, uso de tecnologías inapropiadas, y problemas para comercializar. Se concluye que es necesario atenderlos de manera directa, fortalecer la formación de los técnicos, y aportar a la reorganización de las prácticas de extensión con sentido más participativo y horizontal.

\begin{abstract}
Aiming to identify the problems faced by Latin American rural extensionists, 589 practitioners that work in ten different countries were surveyed. The open questions were categorized following Grounded Theory. The differences between national samples were studied using chi-square. Fifteen problems are highlighted because of having been frequently mentioned including: difficulties in group or cooperative work, use of inappropriate technologies, and problems to sell the produce. It is fundamental to address these problems, to strengthen rural extensionists' training and to re-shape rural extension practices.
\end{abstract}

Palabras clave: extensión rural, América Latina, trabajo asociativo, adopción de tecnologías, participación.

Keywords: rural extension, Latin America, cooperative work, adoption of technologies', participation.

* Doctor en Psicología por la Universidad de Buenos Aires. Adscrito al Consejo Nacional de Investigaciones Científicas y Técnicas (CONICET). Académico en la Universidad de la Cuenca del Plata y la Universidad de Morón. 


\section{Introducción}

- 1 a extensión rural (ER) está retornando en América Latina (Aguirre, 2012; Alemany $\&$ Sevilla, 2007), después del auge privatizador vivido en la mayor parte del subcontinente hacia fines del siglo $\mathrm{xx}$, en el contexto de las políticas neoliberales (Diesel, Froehlich, Neumann \& Silveira, 2008; Rivera, 2002). Se prefiere hablar de ER y no de comunicación para la innovación rural (Leeuwis, 2004), o de comunicación rural (Freire, 1973), ya que se trata de la referencia más utilizada en América Latina. Esto no significa sostener una concepción tradicional de ER, sino utilizar la noción para nombrar el trabajo realizado por agentes de desarrollo rural, el cual puede ir desde la tradicional transferencia de tecnologías, hasta el apoyo a procesos de articulación institucional en ámbitos rurales orientados a fomentar dinámicas de innovación.

En el contexto del regreso de la ER en América Latina, y reconociendo el importante papel que puede jugar en los procesos de desarrollo rural (Selis, 2012; Omar, Bakar, Jais \& Ibaraik, 2011; Zwane, 2012), resulta fundamental producir información empírica que ayude a comprender los problemas a los que se enfrenta, y generar alternativas para resolverlos. No obstante, la mayoría de los trabajos que abordan la ER suelen ubicarse en tres tipos. El primero reúne textos que discuten conceptualmente qué es la ER o cómo se la debería enfocar. Se trata de análisis teóricos que suelen tener escaso soporte en la investigación, aunque son esenciales para discutir la noción misma de ER y sus modelos de práctica. Aun así, no deben entenderse como productos de investigación. A este grupo pertenece el clásico Extensión o Comunicación de Freire (1973). Por otro lado, existe un amplio conjunto de estudios de caso diversos, relacionados con prácticas, instituciones e iniciativas de ER (e.g., Gaitán \& Pachón, 2010; Selis, 2012; Zuin, Zuin \& Manrique, 2011). Finalmente, se encuentran abundantes trabajos institucionales o artículos de opinión de expertos que presentan problemáticas, desafíos y propuestas para el trabajo de ER, usualmente apoyados en datos estadísticos o reflexiones sobre casos y experiencias (e.g., Aguirre, 2012; Ardila, 2010; McLeod \& Qamar, 2003).

Aun reconociendo la vital importancia de tales trabajos, cabe señalar la limitada disponibilidad de literatura que analice la problemática de la ER en América Latina desde una perspectiva amplia (que rebase los estudios de caso), que a la vez haya surgido de una investigación. Así, en este artículo se presentan los resultados de una investigación realizada a partir de encuestas a extensionistas rurales que trabajan en diferentes países de América Latina, con el propósito de identificar los problemas vinculados con el desarrollo de los pequeños productores y las dificultades que encuentran en el trabajo de ER con ellos. Sin 
embargo, antes se presentan los problemas relativos a la ER que se han identificado en la literatura.

\section{Problemas para el desarrollo y la extensión en la bibliografía}

Los problemas identificados en la literatura sobre el desarrollo rural y el trabajo de ER son múltiples, pero dentro de esta multiplicidad se observan diferentes concepciones referidas a los fines de la ER, lo que deriva en que ciertas cuestiones pueden verse como problemas y otras no. Por ejemplo, la falta de adopción de tecnologías por parte de los productores ha sido señalada como un problema central, mientras otro autor, frente a la misma situación, destaca la falta de adecuación de las propuestas de los técnicos a la realidad de los productores. Así, la enunciación de un determinado problema habla tanto de su existencia como del modo de pensar de quien lo considera como tal. A continuación, se presentan los problemas hallados en la bibliografía, que se relacionan con la dificultad de los campesinos o agricultores familiares para mejorar su nivel de vida, y aquellos con los que lidian los extensionistas cuando trabajan aquéllos. Se procede de este modo por dos razones. Primero, porque esos dos problemas no aparecen diferenciados con claridad en la bibliografía, y segundo, porque, como se explicará en la metodología, los propios extensionistas encuestados no diferencian entre ambos. El orden de presentación no implica mayor importancia ni refiere a mayor frecuencia de aparición. Asimismo, debido a que muchas de las referencias utilizadas aquí corresponden a estudios de caso, no son generalizables al conjunto de América Latina.

Un problema que aparece con frecuencia en la literatura son las (1) prácticas productivas inapropiadas de los productores y la no adopción de tecnologias. Hay estudios que observan que un bajo rendimiento de cultivos se debe a prácticas inadecuadas (Lacki, 2006), o al desconocimiento de nuevas tecnologías (Galindo, Pérez, López \& Robles, 2001). Además, se alude al deterioro de los suelos por sobrepastoreo, lo que conduce a bajos rendimientos (Miranda et al., 2011). O bien, de inadecuado manejo y alimentación de animales (Campos et al., 2005), entre más cuestiones. Ante esto, se han identificado el desinterés, la no aceptación, o incluso cierta resistencia, por parte de los productores a la asistencia técnica (Henz, 2010; Salvador, 2010). Esta situación ha sido descrita en términos de falta de adopción de tecnologías (Boas \& Goldey, 2005; Gadea, 2009; Hosseini \& Soltani, 2011). Adicionalmente, otras investigaciones argumentan que lo descrito se agrava por el (2) bajo nivel educativo de muchos productores, lo cual dificulta la comprensión de la información y los argumentos técnicos (Gaitán \& Pachón, 2010; Henz, 2010). 
También se encuentran referencias a la (3) pasividad y/o falta de participación de los productores en distintos proyectos e iniciativas. En este sentido, Boas \& Goldey (2005) refieren diferentes proyectos con dificultades para lograr la participación de los beneficiarios, mientras que Landini (2010) muestra cómo los productores pueden posicionarse pasivamente a la espera de ayudas en contextos clientelares. Cuando se discute la falta de participación o la pasividad de los beneficiarios, las investigaciones argumentan que se debe a que ellos no participaron en el diseño de los programas para su apoyo (Rivas, Avendaño \& Quintero, 2010; Saraiva \& Callou, 2009). Esto se vincula a que (4) las planificaciones no siempre se ajustan a la situación, características o necesidades de los productores que se busca beneficiar. Así, por ejemplo, proyectos de crédito orientados a destinatarios que no pueden cumplir con los requisitos establecidos (Aquino \& Teixeira, 2005), o acciones de extensión que no responden a las expectativas de su público (Murillo \& Martínez, 2010; Taveira \& Oliveira, 2008).

En la literatura también se refiere los (5) problemas para la comercialización que enfrentan los agricultores familiares (Ferrer, Silvetti, Cáceres \& Soto, 2006), en particular, los de los productores no-estructurados (Silva y Leitão, 2009). Ello se explica, muchas veces, por no alcanzar la calidad que exige el mercado o por los bajos volúmenes de producto (Escobar, 2012). En este sentido se ha sugerido la importancia de implementar estrategias asociativas (cooperativas, por ejemplo) para mitigar o superar la falta de volumen en la producción. No obstante, existe (6) un escaso nivel de organización, asi como problemas de los productores para trabajar de modo asociado (Landini, 2007). Esto se explica, entre otras razones, por la orientación individualista de los productores (Boas \& Goldey, 2005).

Otro problema recurrente en la literatura revisada es la (7) implementación de estrategias o enfoques de extensión transferencistas o productivistas, en tanto prácticas de extensión inapropiadas. Por ejemplo, Sánchez (2011) y Saraiva \& Callou (2009) señalan que la extensión tiende a implementarse de modo directivo, vertical y unidireccional, situación que — argumentan otros autores — lleva a desconocer, rechazar o desentenderse de los saberes populares (Rivas et al., 2010; Turiján, Damián, Ramírez, Juárez \& Estrella, 2012). Se trata, en definitiva, de la puesta en práctica de un modelo de extensión difusionista, orientado a promover una agricultura "moderna", pese a que en lo institucional se hable de un modelo participativo o territorial (Selis, 2012), alejado del difusionismo. En paralelo, otros autores son críticos frente a la implementación de un modelo de extensión productivista, es decir, tan focalizado en la dimensión productiva que no considera los factores sociales (Almeida, Oliveira \& Xavier, 2010; Zuin et al., 2011). En este sentido, Rodríguez (2009) afirma que la supuesta "falta 
de adopción de tecnologías" puede también entenderse en términos de la implementación de una estrategia de ER que no toma en cuenta a la gente, a sus expectativas y a sus conocimientos.

Asimismo, otros estudios han destacado la (8) debilidad o falta de cobertura de las instituciones de ER. Esto se explica por la falta de presupuesto para las actividades de extensión (Landini, 2012a; Rivera, 2002), y por la falta de personal para ejecutarlas (Aquino \& Teixeira, 2005; Salvador, 2010). Por su parte, Sepulcri \& Paula (2008) hablan de fragilidad del sistema de extensión, mientras que Ardila (2010) de desmantelación y debilitamiento, situación que dificulta el acceso a la asistencia técnica de los productores con menos recursos.

Otras investigaciones observan el problema de la (9) falta de políticas apropiadas de desarrollo y ER. Miranda et al. (2011), por ejemplo, dan cuenta de la falta de una propuesta integradora y sistemática de desarrollo; Domit, Dalbosco, Santos \& Guimarães (2008), discuten la falta de una política de transferencia de tecnología, y Hosseini \& Soltani (2011) abordan la existencia de una política de desarrollo rural inadecuada.

Concentrándose en los extensionistas rurales, varios autores han marcado como problema (10) la inestabilidad o fragilidad de la situación laboral, por ejemplo, cuando a los extensionistas rurales se les contrata por un tiempo específico, esto es, sin integrarlos a la planta permanente (Ardila, 2010), o cuando se les sujeta a malas condiciones laborales (Sánchez, 2011). Al mismo tiempo se ha indicado la (11) falta de formación apropiada de los extensionistas, con lo que se hace referencia a una formación tecnicista o productivista poco útil para trabajar con productores de baja escala y escasa disponibilidad de capital (Lacki, 2006; Méndez, 2006; Thornthon, 2009). Y las limitaciones en la formación de quienes trabajan en extensión ciertamente contribuye a la existencia de (12) problemas de comunicación con los productores, debido a la existencia de una importante distancia cultural (Landini \& Murtagh, 2011), la cual se nutre de la soberbia de los profesionales que se sienten dueños del saber y rechazan el de las comunidades (Zuluaga, 1994).

También es posible encontrar un conjunto amplio de (13) otros problemas, que sólo se refieren de modo esporádico en la bibliografía. Entre éstos se encuentran la politización de la ER, a través del nombramiento de extensionistas por vínculos políticos o de la selección de productores con fines electorales (Lacki, 2002; Landini, 2012a; Rivera, 2002); los escasos recursos de que disponen los agricultores familiares (Aquino \& Teixeira, 2005; Gaitán \& Pachón, 2010) y la dificultad para conformar equipos interdisciplinarios en el ámbito de la extensión (Sánchez, 2011; Thornthon, 2009).

En resumen, la literatura ha tratado un conjunto amplio de problemáticas vinculadas con el trabajo de extensión y con el desarrollo de la agricultura 
familiar en los países latinoamericanos. Veamos ahora los resultados de la investigación implementada con el fin de cuantificar, desde el punto de vista de los extensionistas, la importancia relativa de estos problemas y las diferencias existentes entre las muestras de los diferentes países.

\section{Metodología}

A partir de la realización de una encuesta (Montero \& León, 2007) se realizó una investigación cuali-cuantitativa, fundamentalmente descriptiva, de carácter transversal, orientada a reconstruir la perspectiva de los extensionistas que trabajan en diez países de América Latina. Tales países fueron Argentina, Bolivia, Brasil, Chile, Ecuador, El Salvador, México, Paraguay, Perú y Uruguay. Luego de construida, la encuesta fue evaluada junto a un pequeño grupo de extensionistas rurales argentinos, lo que llevó a hacerle pequeńas modificaciones de formato y redacción. Asimismo, en cada nuevo país en que se aplicó, fue revisada por un técnico local, para evitar diferencias en el uso del vocabulario. La investigación que enmarca este artículo excede los objetivos propuestos en ese trabajo. La encuesta se estructuró en dos grandes partes, una de preguntas cerradas sociodemográficas, y otra con diferentes preguntas abiertas. De estas últimas se analizan en este trabajo sólo las dos primeras. La redacción estándar de esas preguntas fue como sigue:

1. Según su experiencia, ¿cuáles son los principales problemas que tienen los pequeños productores para progresar y salir adelante? Puede incluir problemas de toda clase (sociales, psicológicos, económicos, culturales, productivos, políticos, institucionales, etc.).

2. Muchas veces los proyectos de extensión y de desarrollo rural con pequeños productores no logran los resultados que se esperan. Además de lo mencionado en la pregunta anterior, ¿qué otras dificultades surgen en el trabajo de extensión? En su respuesta puede incluir dificultades de toda clase (sociales, psicológicas, económicas, culturales, productivas, políticas, institucionales, etc.).

Para enviar la encuesta se contactó con distintas instituciones que trabajan en ER, sobre todo públicas cuando éstas existían y eran accesibles. Cuando esta gestión no dio resultados, se buscó el apoyo de organizaciones no gubernamentales (ONG) de desarrollo rural que realizaran tareas de extensión, o de investigadores y profesionales vinculados al tema. La encuesta fue enviada por correo electrónico a las autoridades y personas que manifestaron voluntad de 
cooperar, éstas la reenviaron a extensionistas pidiendo su colaboración. Las respuestas fueron voluntarias y se recibieron por correo electrónico. En la mayoría de los casos, la respuesta se nos envió directamente, aunque en ocasiones las autoridades institucionales correspondientes centralizaron la recepción. Se recibieron 589 encuestas procesables durante 2010, 2011 y 2012. En la tabla 1 se desglosa la cantidad de sujetos encuestados por país, divididos por sexo, más el porcentaje con educación universitaria completa, su área de formación y su edad media. En la formación universitaria se diferencia entre la "técnica" (ingenieros agrónomos, veterinarios y zootecnistas), la "social" (sociólogos, comunicadores sociales, antropólogos y similares) y "otra" (biólogos, licenciados en ciencias ambientales o recursos naturales $\mathrm{u}$ otras no incluida en las anteriores).

Tabla 1. Características generales de las muestras

\begin{tabular}{|c|c|c|c|c|c|c|c|c|}
\hline \multirow[b]{2}{*}{ País } & \multirow[b]{2}{*}{$n=$} & \multicolumn{2}{|c|}{ Sexo } & \multirow{2}{*}{$\begin{array}{c}\text { Con título } \\
\text { universitario \% }\end{array}$} & \multicolumn{3}{|c|}{ Formación universitaria* } & \multirow{2}{*}{$\begin{array}{l}\text { Edad } \\
\text { Media }\end{array}$} \\
\hline & & $\begin{array}{c}\text { Masculino } \\
\%\end{array}$ & $\begin{array}{c}\text { Femenino } \\
\%\end{array}$ & & $\begin{array}{c}\text { Técnica } \\
\%\end{array}$ & Social \% & Otra \% & \\
\hline Argentina & 220 & 65 & 35 & 84.8 & 81.5 & 9.2 & 9.2 & 42.1 \\
\hline Bolivia & 19 & 68.4 & 31.6 & 85.4 & 68.6 & 11.4 & 20 & 41.4 \\
\hline Brasil & 52 & 59.6 & 40.4 & 94.1 & 75.0 & 12.5 & 12.5 & 45.8 \\
\hline Chile & 41 & 46.3 & 53.7 & 88.9 & 87.5 & 0 & 12.5 & 40.1 \\
\hline Ecuador & 74 & 73.6 & 26.4 & 90.3 & 90.8 & 0 & 9.2 & 37.0 \\
\hline El Salvador & 34 & 97.1 & 2.9 & 77.4 & 95.8 & 0 & 4.2 & 41.3 \\
\hline México & 60 & 73.3 & 26.7 & 91.8 & 75.6 & 6.7 & 17.8 & 41.1 \\
\hline Paraguay & 26 & 72 & 28 & 60.9 & 71.43 & 0 & 28.57 & 34.4 \\
\hline Perú & 31 & 90.3 & 9.7 & 93.1 & 85.2 & 0 & 14.8 & 39.8 \\
\hline Uruguay & 32 & 54.8 & 45.2 & 93.6 & 62.1 & 37.9 & 0 & 40.3 \\
\hline Total/Media** & 589 & 70.0 & 30.0 & 86.0 & 79.3 & 7.8 & 12.9 & 40.3 \\
\hline
\end{tabular}

Para identificar, cuantificar y comparar los problemas que enfrentan los extensionistas rurales de distintos países, primero se incorporaron las encuestas al programa Atlas Ti. Se decidió analizar en conjunto las respuestas a las dos preguntas de la encuesta, porque los encuestados tendían a no diferenciar los problemas de los productores de los que surgen en el trabajo de ER con ellos. Utilizando el Atlas Ti, se procedió a la lectura de las respuestas, que fueron ordenadas a partir de la construcción de categorías, utilizándose los principios de la teoría fundamentada (Glaser \& Strauss, 1967). Las categorías, es decir, los distintos problemas que aparecían, se delimitaron de modo progresivo a partir de por lo 
menos cuatro lecturas de las respuestas. En ese proceso se construyeron definiciones precisas de cada categoría, a las cuales se incorporaron criterios para incluir o excluir fragmentos ante situaciones dudosas. Hasta que las categorías estuvieron definidas, los casos dudosos fueron categorizados. Luego se procedió a leer todos los fragmentos incluidos en las distintas categorías, excluyéndose los que no correspondían a su definición final. Frente a categorías con límites difusos, se optó por unirlas. Pero si los límites de una categoría generaban dudas constantes sobre la inclusión o exclusión de fragmentos, incluso en esa etapa final, se optó por considerar que la categoría no era pasible de análisis cuantitativo.

Seguidamente, los resultados del Atlas Ti se trasladaron al software SPSS 17.0. Para esto se consideró a cada categoría construida como una variable. En total se construyeron 83 categorías de problemas, en su mayoría consideradas como cuantificables. Para proceder a la cuantificación, se consideró que un problema estaba presente en una encuesta cuando el encuestado lo había mencionado al menos una vez. De esta manera, las categorías fueron tomadas como variables nominales dicotómicas, cuyos valores fueron presencia (si el encuestado la había mencionado) o ausencia (si no lo había hecho). Dada la amplitud de categorías y la presencia desigual en los distintos países, se decidió presentar las más relevantes. Para esto, se excluyeron primero los problemas considerados no cuantificables, para luego establecer como criterio de relevancia el cumplimiento de al menos una de las siguientes pautas: el problema había sido mencionado por el $30 \%$ o más de los encuestados de un país; por el $25 \%$ o más de dos países; por el $20 \%$ o más de tres países; o la media de referencias al problema entre todos los países era de $15 \%$ o más. Cuando un problema no cumplía estos requerimientos, se excluía del listado de problemas que serían analizados. Para conocer si estos problemas se distribuían de manera desigual entre los diferentes países, se utilizó el estadístico chi cuadrado, sin que haya casos en los cuales el $20 \%$ de las casillas o más tuvieran un valor esperado menor a cinco.

\section{Resultados y discusión}

En la tabla 2 se presentan los principales problemas para el trabajo de ER y para el desarrollo de la agricultura familiar que se han detectado. En esa tabla los problemas se han ordenado a partir del promedio de las medias de las muestras nacionales. También se analiza si existen diferencias estadísticamente significativas entre países utilizando chi cuadrado. 


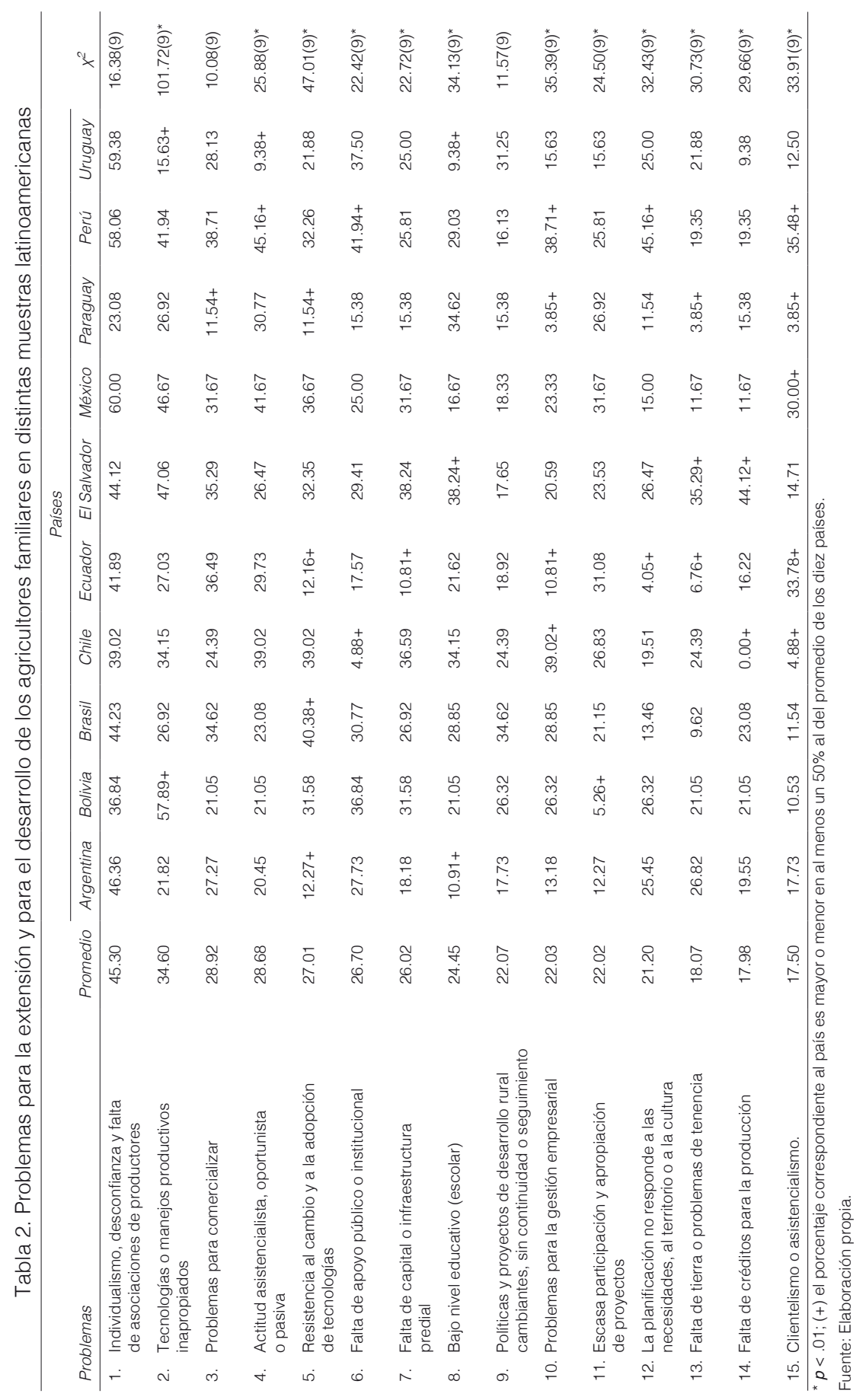


El problema que en promedio fue más mencionado es el individualismo, la desconfianza y la falta de asociaciones de productores (45.3\%), citado también con cierta frecuencia en la literatura, aunque en ninguno de los casos se le ubica como problemática fundamental, como aparece aquí. La importancia de esta área problemática reclama la implementación de una estrategia de ER concentrada en la prestación de apoyo a grupos y no sólo a individuos (Escobar, 2012), propuesta que considera la importancia del trabajo asociativo en la reducción de costos para la compra de insumos y el fortalecimiento de la capacidad de comercialización (Elgue \& Chiaradía, 2007). Asimismo, la preocupación por esta problemática parece relacionarse con el perfil formativo de los extensionistas, en su gran mayoría provenientes de áreas técnicas (Landini \& Bianqui, 2014; Thornthon, 2009), lo que revela su escasa formación para la gestión de procesos sociales como el trabajo grupal o asociativo (Landini, Murtagh \& Lacanna, 2009).

Luego, con una media de $34.6 \%$, aparecen los manejos productivos inadecuados y la falta de tecnologías modernas entre los productores, lo que se complementa con el quinto problema, alusivo a la resistencia al cambio y a la adopción de tecnologías e indicado con un promedio del $27 \%$. Estos dos problemas, omnipresentes en la bibliografía sobre la temática, sugieren una concepción de extensión en la cual la dimensión tecnológica y la transferencia de tecnologías ocupan un lugar central, lo que expresaría una extensión productivista/ difusionista. Esto abona a las críticas que son parte significativa de la literatura referida a la existencia de prácticas de ER tradicionales, centradas en la transferencia de tecnologías. Esto, además, estaría en línea con la formación predominantemente tecno-productiva de los extensionistas. Ahora bien, si desde esta mirada crítica de la ER se puede cuestionar la supuesta "resistencia a la adopción", argumentando que se debe a la falta de comprensión de la racionalidad del agricultor familiar (Landini, 2011), no se debe olvidar la importancia que tiene para los extensionistas como problema sensible. Además, se trata de una dimensión de la extensión (la dimensión informativa), que no debe excluirse al ampliar y complejizar su concepción, sino que debe servir para repensarla en un contexto diferente.

En tercer lugar, una media del $28.9 \%$ de los extensionistas de los distintos países indica problemas para comercializar la producción. Así, esta cuestión viene a ser fundamental. Cuando se abordan, los problemas de comercialización se trabajan a partir de la cooperativización (fortalecimiento de la capacidad de negociación) de los productores (Ferrer et al., 2006; Henz, 2010). Sin embargo, no deben olvidarse las otras alternativas para el apoyo de la comer- 
cialización, como las compras públicas de productos de la agricultura familiar (Zaar, 2011) o el respaldo que se da a los agricultores para que se articulen a la cadena comercial.

Una actitud asistencialista, oportunista o pasiva entre los productores ha sido mencionada como problema por un promedio de $28.7 \%$ de las muestras, clasificándolo así como un problema relevante. En cierto sentido, éste tal vez derive de la práctica de estrategias o metodologías de extensión difusionistas, o que se desentienden o no responden a las necesidades de los productores, inconveniente indicado también por una media del $21.2 \%$. En efecto, será difícil contar con la participación de los beneficiarios si las propuestas no se corresponden con las necesidades. La actitud asistencialista o pasiva de los productores se articula como problema de manera constitutiva con aquel descrito como escasa participación y apropiación de los proyectos por parte de los productores, señalado por una media de $22 \%$ de los integrantes de las muestras latinoamericanas. Al pensar en ambos al mismo tiempo, es decir, la preocupación por la falta de participación e implicación de los productores, así como por la adopción de posturas pasivas o asistencialistas, debe considerarse la existencia de condiciones históricas y sociales que a lo largo del tiempo no han favorecido (ni permitido) un posicionamiento activo de los sectores campesinos latinoamericanos (Landini, 2012b; Martín-Baró, 1987). Esto sin duda dificulta la participación de los beneficiarios, al menos en un inicio. Por otra parte, la mención de estos problemas también debe insertarse en el proceso actual de reorientación en términos participativos de muchas instituciones e iniciativas de ER (Da Ros, 2012; Escobar, 2012; Gutiérrez, 2010; Rodríguez, 2009), un dominio en el que el personal de extensión, mayormente formado en áreas técnicas, se autopercibe con dificultades para gestionar (Landini, Murtagh \& Lacanna, 2009), lo que lleva de nuevo a los citados problemas de formación de los extensionistas. Así, los extensionistas percibirían falta de participación y pasividad, en especial porque la primera se convierte en un requisito de su práctica.

En sexto lugar, mencionado por un promedio de $26.7 \%$ de las muestras, aparece el problema de la falta de apoyo público o institucional a los productores, lo que se complementa con la ausencia de políticas de desarrollo rural que tomen en cuenta la agricultura familiar, un asunto relacionado con las políticas neoliberales implementadas en América Latina en las últimas dos décadas del siglo xx, que han propuesto el retiro de los Estados de la economía, así como políticas de ajuste estructural que han apoyado la privatización de los servicios de ER en la región (Ardila, 2010; Diesel et al., 2008; Rivera, 2002).

Sin perder el foco en las políticas públicas, los resultados muestran que una media del $22.1 \%$ afirma que las políticas, proyectos e iniciativas de desarrollo 
rural son cambiantes, discontinuas y les falta seguimiento. Esto lo explican, por un lado, a partir del relevo de autoridades institucionales, que lleva a cambiar las políticas de gobierno cada vez que asume un nuevo presidente, ministro o autoridad de la institución de extensión. Por el otro, que a los proyectos, una vez ejecutados, no se les da el seguimiento apropiado. Esto permite observar que las políticas de desarrollo son iniciativas de gobierno y no de Estado, es decir, que son llevadas adelante por los sectores en el poder, pero sin responder a acuerdos más amplios con diferentes actores implicados o interesados.

En las encuestas se identificaron otros problemas como la falta de capital, inversión o infraestructura predial en las fincas de los productores (26\%); un tema ya tratado en la literatura sobre la ER. Para Carmagnani (2008) ello es un factor característico que diferencia entre agricultura familiar y empresarial en los distintos países latinoamericanos. Otro más es el bajo nivel educativo de los productores $(24.4 \%)$, situación que sugiere que el desarrollo económicoproductivo rural no puede desentenderse de otros aspectos como la educación, lo que defiende con vehemencia Lacki (2002). No obstante, la preocupación por el bajo nivel educativo también puede indicar una dificultad para implementar estrategias pedagógicas y formas menos teóricas de comunicarse con los productores. Es decir, más adecuadas a su formación escolar.

Los problemas para la gestión empresarial $(22 \%)$ aluden a que los beneficiarios no gestionan su actividad como un negocio o empresa, analizando gastos e ingresos y llevando registros, sino que lo hacen más por intuición, donde el afán de lucro convive con otros intereses al tomar decisiones (Landini, 2011). Que se identifique la falta de gestión empresarial como problema muestra la voluntad de convertir a los productores en empresarios como parte de la propuesta de ER.

Luego está la problemática de la falta de tierra (superficies muy pequeñas o inapropiadas para la producción) o los problemas legales de tenencia (18.1\%), algo que llamativamente no se menciona como problema actual en la literatura consultada, aun cuando pueda ser una cuestión acuciante en muchos contextos y genere importantes conflictos sociales. Después se ubica la cuestión de la falta de créditos para la producción (18\%), tema presente en la revisión bibliográfica, lo que se vincula a la falta de capital e infraestructura predial indicada más arriba. Finalmente, con el $17.5 \%$ de menciones en promedio en los distintos países, se ubica la implementación de políticas o estrategias de desarrollo y ER de carácter clientelar, asistencialista o paternalista, hecho que constituye, en algún punto, la contracara del problema descrito como actitud asistencialista, oportunista o pasiva por parte de los productores, en tanto las prácticas clientelares/asistencialistas pueden inducir a estrategias de subsistencia pasivas (Landini, 2012b). 
Respecto de la mayor parte de los problemas descritos, se observan diferencias estadísticamente significativas entre los países. Abajo se exponen los casos en los que la media de referencias a cada problema en un país determinado fue al menos $50 \%$ superior o $50 \%$ inferior a la media de todos los países (pero sólo cuando las diferencias entre éstos hayan probado ser estadísticamente significativas).

1. Argentina. Se caracteriza por menos referencias a la resistencia al cambio de los productores y a su bajo nivel educativo. Esto sugiere mayor nivel educativo de los productores en general, así como un perfil de extensionista posiblemente menos difusionista (Landini, 2015), al señalarse menos la resistencia al cambio y la falta de adopción como un problema.

2. Bolivia. Los encuestados se caracterizan por una actitud bastante más crítica que la media respecto de las tecnologías y manejos productivos de los beneficiarios, lo que sugiere una mirada que valora menos el saber y la perspectiva de la gente. En cambio, la referencia a la escasa participación y apropiación de proyectos es significativamente menor que la media, por lo cual debe ponerse en entredicho la existencia de un "rechazo de la perspectiva del productor" en la muestra boliviana, ya que, si así fuera, sería esperable encontrarse con más problemas referidos a la falta de participación.

3. Brasil. Más del 40\% de los encuestados de Brasil reportan resistencia al cambio y a la adopción de tecnologías por parte de los productores, lo que representa un $50 \%$ más que la media de los distintos países. Esto obliga a preguntarse si las prácticas de extensión en Brasil tendrán un formato más transferencista que en otros países latinoamericanos. De ser así, resultaría llamativo, ya que Brasil ha establecido como política pública nacional un modelo de extensión participativo y agroecológico (Medeiros, 2007).

4. Chile. Las diferencias en los encuestados de Chile respecto de la media general sugieren la existencia de un perfil particular, tal vez marcado por una perspectiva neoliberal, característica en el país en las últimas décadas. En este sentido, son pocos los encuestados que hablan de falta de apoyo público para el desarrollo y la extensión, lo que podría deberse ya sea a un apoyo fuerte del Estado al desarrollo rural o, lo más probable, a la existencia de menores requerimientos por parte de los extensionistas con respecto a apoyos y compromisos desde el sector público con el desarrollo. A esto se suma una gran cantidad de referencias a problemas con la gestión empresarial de parte de los productores, 
algo que en Chile es una preocupación fundamental. Por otra parte, ningún encuestado refiere problemas con la obtención de créditos, posiblemente por la facilidad para obtenerlos y, finalmente, son pocos los que hablan de prácticas políticas clientelares o de acciones de tipo asistencialista o clientelar.

5. Ecuador. En seis problemas se encuentran diferencias de más del 50\% (hacia arriba, o hacia abajo) respecto de la media. Por un lado, parece observarse una menor orientación difusionista de los encuestados ecuatorianos, ya que se habla con menos frecuencia de resistencia al cambio y de falta de adopción de tecnologías. A la vez, se menciona muy poco que las planificaciones entran en contradicción con las necesidades, características y contexto de los productores, lo que sugiere un enfoque más participativo. Asimismo, se citan con menor frecuencia problemas de falta de capital e infraestructura predial, y de falta de tierra para la producción. A la vez, el problema de la falta de gestión empresarial del productor es señalado en menos oportunidades, lo que hace pensar que la conversión del productor en un empresario no aparece como prioridad, mientras que sí destaca la implementación de acciones de extensión de carácter asistencialista, fundamentalmente por parte de ONG.

6. El Salvador. En El Salvador se encuentran referencias más frecuentes al bajo nivel educativo de los productores, a problemas con la tierra y al limitado acceso a créditos para la producción.

7. México. La muestra mexicana sólo destaca porque los encuestados señalan en mayor número que la media tiene problemas con prácticas de tipo clientelar o asistencialista por parte de gobiernos e instituciones.

8. Paraguay. Los datos de Paraguay son llamativos, ya que en los cuatro casos que presentan diferencias respecto de la media, éstas son hacia abajo. Es decir, los encuestados mencionan con mucha menor frecuencia tener problemas de resistencia al cambio y a la adopción de tecnologías, encontrarse con problemas para la gestión empresarial, enfrentarse a problemas de tierras u observar prácticas clientelares o asistenciales por parte de gobiernos e instituciones. Ahora bien, dado que los extensionistas paraguayos tuvieron respuestas bastante más cortas que la media en general, se prefiere señalar estos resultados manteniéndose cautos respecto de las interpretaciones posibles, ya que es probable que esta menor incidencia sea por una menor extensión de las respuestas.

9. Perú. Los encuestados peruanos señalan con mayor frecuencia que la media que encuentran actitudes pasivas, oportunistas o asistencialistas de los productores, 
lo que claramente se complementa con más del doble de referencias al problema de que la planificación que se realiza desde las instituciones no corresponde a las necesidades y características de los productores. Resulta claro, cuanto menos respondan las acciones de extensión a las necesidades de los productores, más posicionamientos pasivos y actitudes oportunistas se presentarán. Por otra parte, la falta de una mentalidad empresarial que conciba la actividad productiva como negocio se menciona en un alto porcentaje de los encuestados, como sucedía en Chile. Finalmente, también se encontró una mayor preocupación por la falta de apoyo público o institucional para el desarrollo y la ER, así como mayores reclamos por la existencia de prácticas políticas clientelares y por la implementación de iniciativas con fuerte tinte asistencialista o paternalista.

10. Uruguay. El caso de Uruguay resulta muy interesante. En primer lugar, se observan pocas dificultades causadas por el bajo nivel escolar de los beneficiarios, y Uruguay es un país con muy buenos indicadores educativos en el contexto latinoamericano. Por otra parte, se observan bastante menos referencias que en la media a que los productores poseen prácticas productivas inapropiadas o adoptan actitudes pasivas y/o asistencialistas. Esto sugiere una mirada menos crítica hacia los productores y sus actitudes, lo que puede significar la presencia de una ER más comprensiva del productor, de sus saberes y sus posicionamientos.

\section{Reflexiones finales}

El presente trabajo permite diferentes conclusiones y sugiere distintas líneas de reflexión. En primer lugar, un listado jerarquizado de problemas, tanto como resultado de la articulación de todas las muestras nacionales, como uno para la muestra de cada país, constituye una información valiosa sobre las dificultades que enfrenta la ER en cada contexto, desde el punto de vista de los propios extensionistas. Contar con un instrumento así permite, a falta de otros, medir su importancia relativa y pensar estrategias para un mejor abordaje. E incluso aportar a su visibilización, en caso de que alguno de ellos no se hubiera tomado en cuenta lo suficiente.

El individualismo, la desconfianza y la falta de asociaciones de productores han sido identificados en este estudio como el problema más mencionado, a lo que se suma la importante diferencia cuantitativa respecto de los demás. Así, urge dar a este problema la importancia que merece, lo que implica aportar a su comprensión con investigación e implementar estrategias de acción que contribuyan a enfrentarlo. Éstas deberían contemplar dos niveles al menos: el diseño de proyectos de extensión que aprovechen las ventajas del trabajo grupal 
y asociativo pero minimizando sus problemas, y la construcción de modalidades de trabajo e interacción con los beneficiarios que reduzcan la incidencia y los resultados negativos de este tipo de dificultades.

Un punto imposible de soslayar en este contexto es la formación fundamentalmente técnica de los extensionistas que trabajan en los distintos países (Landini \& Bianqui, 2014), la cual no resulta apropiada para gestionar o apoyar procesos grupales u organizativos. En este sentido es imperioso, primero, avanzar hacia una mayor diversidad disciplinar en el trabajo de ER. Resulta contradictorio contratar sólo ingenieros agrónomos y veterinarios cuando se les pedirá que realicen tareas que exceden su profesión. También resulta fundamental incluir temáticas como el trabajo grupal y asociativo y sus problemas en las capacitaciones que se dan a los extensionistas. Brindar capacitaciones sólo referidas a cuestiones técnicas o comerciales no es la mejor alternativa para contribuir al éxito de las intervenciones (Landini, 2013). Las capacitaciones no deberían abarcar los temas que resultan más intuitivos para los profesionales y para sus autoridades, sino aquellas cuestiones que traen problemas en la práctica. Así, la propuesta de pensar la capacitación de los extensionistas a partir de los problemas de la práctica, que exceden su formación pero que están dentro de su campo de acción, no sólo alude al trabajo grupal o asociativo sino también a temáticas vinculadas con participación, adopción de posicionamientos activos y procesos de cambio e innovación.

En relación a la orientación productivista/difusionista que parece reflejarse en la importancia otorgada a la aplicación de tecnologías "inapropiadas" por parte de los productores, y a la resistencia al cambio y a la adopción de tecnologías, resulta prioritario elaborar estrategias de acción. La implementación de un modelo de estas características puede estar limitando el impacto de la acciones e incrementando problemas como la actitud asistencialista de los productores, su rechazo a propuestas técnicas y una escasa participación y apropiación de proyectos; en tanto que las propuestas no se construyen con base en las necesidades o prioridades de los productores, sino según los supuestos de burócratas. Ante esto, resulta necesario generar estrategias para reorganizar las prácticas de extensión, lo que no se logra sólo cambiando las instituciones de extensión, sino también, y de manera particular, a través de procesos reflexivos y críticos de formación que permitan modificar el modo de trabajar según esquemas más participativos y horizontales. Las propuestas de cambio en los modos de hacer ER que sólo se orientan al nivel cognitivo tienen escasa potencialidad para transformar prácticas tan articuladas con las identidades de los protagonistas (Landini, Bianqui \& Russo, 2013).

Con respecto al problema de la comercialización, es indudable que la cuestión debe ser considerada desde el diseño mismo de las iniciativas y proyectos, 
considerando que la opción de asociar a los productores es compleja y no constituye la única alternativa; existen otras, como el enfoque por cadenas productivas o las compras institucionales.

Por otra parte, es importante al analizar los distintos problemas observar que no todos se encuentran bajo la influencia de los extensionistas y de las instituciones de ER. La falta de apoyo público, la existencia de políticas con escasa continuidad y la limitada escolaridad de los productores, entre otros problemas, son cuestiones de gran importancia que exceden el campo de incidencia de los espacios de extensión. Por este motivo, cuando se piensa en abordar estas cuestiones debe evitarse caer en dos extremos: la omnipotencia de creer que todo puede resolverse y el fatalismo de quien piensa que nada puede hacerse, porque en los dos casos se termina bloqueando los cambios que sí pueden alcanzarse.

Respecto de las diferencias identificadas entre las distintas muestras nacionales, si bien no es posible aquí un análisis en detalle, debe recordarse que pueden permitir adaptar estas propuestas a contextos particulares, donde prime una $\mathrm{u}$ otra problemática. De todas maneras, resulta necesario volver a señalar que dadas las características de las muestras, éstas no pueden ser consideradas representativas de las realidades de los países, sino meros indicadores de interés que ayudan a generar interpretaciones e hipótesis de lo que resulta más importante y adecuado en cada contexto y territorio en particular.

\section{Referencias}

Aguirre, F. (2012). El nuevo impulso de la extensión rural en América Latina. Situación actual y perspectivas. Santiago de Chile: Red Latinoamericana para los Servicios de Extensión Rural.

Alemany, C., \& Sevilla Guzmán, E. (2007). ¿Vuelve la extensión rural? Reflexiones y propuestas agroecológicas vinculadas con el retorno y fortalecimiento de la extensión rural en América Latina. Realidad Económica, 227, 52-74.

Almeida, S., Oliveira, M. \& Xavier, J. (2010). A descentralização da política nacional de ATER: uma experiência nos assentamentos de reforma agrária no noroeste mineiro-Brasil. Sociedade \& Natureza, 22(3), 551-560.

Aquino, J. \& Teixeira, O. (2005). Agricultura familiar, crédito e mediação institucional: A experiência do PRONAF em São Miguel no Nordeste Brasileiro. Cuadernos de Desarrollo Rural, 54, 61-85.

Ardila, J. (2010). Extensión rural para el desarrollo de la agricultura y la seguridad alimentaria: aspectos conceptuales, situación y una visión de futuro. San José: IICA. 
Boas, A. \& Goldey, P. (2005). A comparison on farmers' participation in farmers' organizations and implications for rural extension in Minais Gerais. Organizaçóes Rurais \& Agroindustriais, 7(3), 259-270.

Campos, M., Machado, H., Matías, Y., González, L., Sánchez, S. \& Duquesne, P. (2005). Diagnóstico socioeconómico, ambiental e institucional de una entidad productiva mediante metodologías participativas. Pastos y Forrajes, 28(4), 331-340.

Carmagnani, M. (2008). La agricultura familiar en América Latina. Problemas del Desarrollo, 39(153), 11-56.

Da Ros, C. (2012). Gênese, desenvolvimento, crise e reformas nos serviços públicos de extensão rural durante a década de 1990. Mundo agrario, 25 [edición electrónica].

Diesel, V., Froehlich, J., Neumann, P. \& Silveira, P. (2008). Privatização dos serviços de extensão rural: uma discussão (des)necessária? Revista de Economia e Sociologia Rural, 46(4), $1155-1188$.

Domit, L., Dalbosco, M., Santos, R. \& Guimarães, M. (2008). Transferência de tecnologia para a cultura da soja-a experiência da copacol. Semina: Ciências Agrárias, 29(2), 255-264.

Elgue, M. \& Chiaradía, C. (2007). Formas asociativas para la agricultura familiar: elementos para el análisis funcional y normativo de las distintas formas jurídicas. Buenos Aires: Secretaría de Agricultura, Ganadería, Pesca y Alimentos.

Escobar, G. (2012). Diseño de una agenda de extensión rural latinoamericana para un desarrollo rural inclusivo. Extensión rural con un enfoque participativo y de mercado: hacia un marco conceptual. Santiago de Chile: Centro Latinoamericano para el Desarrollo Rural.

Ferrer, G., Silvetti, F., Cáceres, D. \& Soto, G. (2006). Análisis de dos iniciativas agroindustriales vinculadas con la capricultura en Córdoba (Argentina). Agroalimentaria, 23, 71-83.

Freire, P. (1973). ¿Extensión o comunicación? La concientización en el medio rural. Buenos Aires: Siglo XXI.

Gadea, R. (2009). Extensión rural en el Paraguay: experiencias del proyecto de manejo sostenible de los recursos naturales (PMRN). Revista IICA Uruguay, 2, 8-10.

Gaitán, C. \& Pachón, F. (2010). Causas para la adopción de tecnologías para la renovación de cafetales-Caso El Colegio (Cundinamarca). Agronomia Colombiana, 28(2), 329-336.

Galindo, G., Pérez, H., López, C. \& Robles, A. (2001). Estrategia de comunicación en el medio rural zacatecano para transferir innovaciones agrícolas. Terra Latinoamericana, 19(4), 393-398. 
Glaser, B. \& Strauss, A. (1967). The Discovery of Grounded Theory. Strategies for Qualitative Research. Chicago: Aldine Transaction.

Gutiérrez, O. (2010). Desarrollo de la metodología Innovación Rural Participativa en la zona andina central de Colombia. Agronomía Colombiana, 28(3), 525-533.

Henz, G. (2010). Desafios enfrentados por agricultores familiares na produção de morango no Distrito Federal. Horticultura Brasileira, 28(3), 260-265.

Hosseini, S. \& Soltani, Z. (2011). The Role of Extension in Adopting Solar Energy in Rural Areas Case of Carbon Sequestration Project. American Journal of Agricultural and Biological Sciences, 6(1), 99-104.

Lacki, P. (2002). Lo que piden los agricultores y lo que pueden los gobiernos. Revista Mexicana de Agronegocios, 6(11), 509-513.

Lacki, P. (2006). Si somos tan ricos ¿por qué somos tan pobres?. Revista MVZ Córdoba, 11(1), 691-693.

Landini, F. (2015). Concepción de "extensión rural" de los extensionistas rurales argentinos que trabajan en el sistema público nacional con pequeños productores. Cuadernos de Desarrollo Rural, 12(75), 35-53.

Landini, F. (2013). Necesidades formativas de los extensionistas rurales paraguayos desde la perspectiva de su función, sus problemas y sus intereses. Trabajo y Sociedad, 20, 149-160.

Landini, F. (2012a). Problemas en la extensión rural paraguaya: modelos de extensión en la encrucijada. Cuadernos de Desarrollo Rural, 69, 127-149.

Landini, F. (2012b). Pasividad, dinamismo y percepción de control en la vida campesina. Investigaciones en Psicología, 17(1), 59-79.

Landini, F. (2011). Racionalidad económica campesina. Mundo Agrario, 23 [edición electrónica].

Landini, F. (2010). Ingenieros extensionistas formoseńos desde la mirada de los pequeños productores. Representaciones, expectativas y realidades. Mundo Agrario, 20 [edición electrónica].

Landini, F. (2007). Prácticas cooperativas en campesinos formoseños. Problemas y alternativas. Revista de la Facultad de Agronomía, 27(2), 173-186.

Landini, F. \& Bianqui, V. (2014). Latin American rural extensionists' socio-demographic profile. Ciência Rural, 44(3), 575-581. 
Landini, F., Bianqui, V. \& Russo, M. (2013). Evaluación de un proceso de capacitación para extensionistas rurales implementado en Paraguay. Revista de Economia e Sociologia Rural, 51(1), s009-s030.

Landini, F. \& Murtagh, S. (2011). Prácticas de extensión rural y vínculos conflictivos entre saberes locales y conocimientos técnicos. Contribuciones desde un estudio de caso realizado en la provincia de Formosa (Argentina). Ra Ximhai, 7(2), 263-279.

Landini, F., Murtagh, M. \& Lacanna, C. (2009). Aportes y reflexiones desde la psicología al trabajo de extensión con pequeños productores. Formosa: INTA.

Leeuwis, C. (2004). Communication for rural innovation. Rethinking agricultural extension. Oxford y Wageningen: Blackwell.

Martín-Baró, I. (1987). El latino indolente: carácter ideológico del fatalismo latinoamericano. En M. Montero (Comp.). Psicología política latinoamericana (pp.135-162). Caracas: Panapo.

Mcleod, W. \& Qamar, M. (2003). Agricultural Extension, Rural Development and the Food Security Challenge. Roma: FAO.

Medeiros, J. \& Borges, D. (2007). Participação cidadã no planejamento das açóes da Emater -RN. Revista de Administração Pública, 41(1), 63-81.

Méndez, M. (2006). Los retos de la extensión ante una nueva y cambiente noción de lo rural. Revista Facultad Nacional de Agronomia, Medellín, 59, 3407-3423.

Miranda, T., Machado, H., Suárez, J., Sánchez, T., Lamela, L., Iglesias, J., Suset, A., Pérez, A., Milera, M., Martín, G., Campo, M., López, O. \& Simón, L. (2011). La innovación y la transferencia de tecnologías en la estación experimental Indio Hatuey (Parte I). Pastos y Forrajes, 34(4), 393-412.

Montero, I. \& León, O. (2007). A Guide for Naming Research Studies in Psychology. International Journal of Clinical and Health Psychology, 7(3), 847-862.

Murillo, D. \& Martínez, J. (2010). Comunicación para el desarrollo en México: reflexiones sobre una experiencia en el trópico húmedo. Estudios sobre las Culturas Contemporáneas, 16(31), 201-225.

Omar, J., Bakar, A., Jais, H. \& Ibaraik, F. (2011). A Review Study of the Reorganization of Agricultural Extension Toward Sustainable Agricultural Development. International Journal of Engineering Science and Technology, 3(5), 4358-4366. 
Rivera, F. (2002). Una aproximación al desarrollo rural de Costa Rica. La extensión agrícola: breve síntesis de su evolución y tendencias. Educare, 3, 29-43.

Rivas, A., Avendaño, P. \& Quintero, H. (2010). Updating peasant competencies to mitigate poverty in the Chorti community, Copan (Honduras). Agronomia Colombiana, 28(3), 567-575.

Rodríguez, A. (2009). Síntesis Marco: La extensión rural en el Cono Sur, nuevos desafíos frente a la Sociedad del Conocimiento. Revista IICA Uruguay, 2, 4-5.

Salvador, D. (2010). A modernização da actividade mandioqueira e uso atual do território do agreste potiguar. Mercator-Revista de Geografia da UFC, 9(20), 93-117.

Sánchez Cadena, D. (2011). Dimensión informativa vs. dimensión comunicativa en el concepto de extensión. ¿¿a definición según Swanson en verdad ya está superada?. Ra Ximhai, $7(3), 437-444$.

Sánchez Vidal, A. (1991). Psicología comunitaria. Bases conceptuales y operativas, métodos de intervención. Barcelona: PPU.

Saraiva, R. \& Callou, A. (2009). Políticas públicas e estratégias de comunicação para o desenvolvimento local de comunidades pesqueiras de Pernambuco. Interaçôes, Campo Grande, 10(1), 73-81.

Selis, D. (2012). Análisis de la institucionalidad asociada a los procesos de innovación tecnológica en el sector hortícola del Gran La Plata. Mundo Agrario, 12(24) [edición electrónica].

Sepulcri, O. \& Paula, N. (2008). O Estado e seus Impactos na Emater-Pr. Revista Paranaense de Desenvolvimento, 114, 87-110.

Silva, F. \& Leitão, M. (2009). Extensão rural e floricultura tropical para o desenvolvimento local: a cooperação no processo de inclusão competitiva dos agricultores familiares em Pernambuco. Interaçôes, Campo Grande, 10(1), 9-19.

Taveira, L. \& Oliveira, J. (2008). A extensão rural na perspectiva de agricultores assentados do Pontal do Paranapanema. Revista de Econonomia e Sociologia Rural, 46(1), 9-30.

Thornthon, R. (2009). Nuevos arreglos institucionales para la extensión rural. Revista IICA Uruguay, 2, 24-25.

Turiján, T., Damián, M., Ramírez, B., Juárez, J. \& Estrella, N. (2012). Manejo tradicional e innovación tecnológica en cultivo de maíz en San José Chiapa, Puebla. Revista Mexicana de Ciencias Agricolas, 3(6), 1085-1100.

F. Landini | Problemas de la extensión rural en América Latina Perfiles Latinoamericanos, 24(47) | FLACso México | pp. 47-68 | DoI: 10.18504/pl2447-005-2016 
Zaar, M. (2011). Las políticas públicas brasileñas y la agricultura familiar: quince años del Programa Nacional de Fortalecimento da Agricultura Familiar (PrONAF). Scripta Nova, 15(351) [edición electrónica].

Zuin, L., Zuin, P. \& Manrique, M. (2011). A comunicação dialógica como fator determinante para os processos de ensino-aprendizagem que ocorrem na capacitação rural: um estudo de caso em um órgão público de extensão localizado no interior do Estado de São Paulo. Ciência Rural, 41(5), 917-923.

Zuluaga, L. (1994). Una aproximación a la investigación participativa. Agronomía Colombiana, 11(1), 107-117.

Zwane, E. (2012). Does Extension Have a Role to Play in Rural Development? South African Journal of Agricultural Extension, 40, 16-24.

Recibido el 8 de mayo de 2013. Aceptado el 16 de junio de 2015. 